\title{
Multiscale modeling and failure analysis of an 8-harness satin woven composite
}

\author{
Zhengmao Yang ${ }^{\mathrm{a}, *}$, Han Yan $^{\mathrm{b}}$ \\ ${ }^{\mathrm{a}}$ Institute of Mechanics, Chinese Academy of Sciences, Beijing, China \\ ${ }^{\mathrm{b}}$ School of Aerospace Engineering, Tsinghua University, Beijing, China
}

\section{A R T I C L E I N F O}

\section{Keywords:}

8-Harness satin weave composites

Failure analysis

Finite element analysis

Mechanical properties

Multi-scale approach

\begin{abstract}
A B S T R A C T
8-harness satin (8HS) woven composites display complex architecture and a certain feature of mutually orthogonal reinforcement, which arise particular interest for aerospace structural groups. In this study, based on realistic geometry parameters and material property, multiscale finite element models of an 8HS woven composite are established. The effective properties of fiber bundles can be obtained by the micro-scale model and then used in the meso-scale model. The meso-scale model is established to predict the global failure behavior of 8HS woven composites under tensile load using the periodic boundary conditions. The predict results are compared to experiments from tensile tests and microstructure analysis on oxide/oxide 8HS woven ceramic matrix composites. The good correlation of the comparison shows that the multiscale modeling approaches can accurately predict the mechanical properties and failure mechanism of $8 \mathrm{HS}$ woven composites under plane tensile load when considering the particular material features.
\end{abstract}

\section{Introduction}

Woven composites, especially the 8HS woven composites, are widely employed in the aerospace industry attributed to higher resistance to impact damage as well as better manufacturing flexibility compared to unidirectional composites [1-3]. As shown in Fig. 1, the unique geometric feature for $8 \mathrm{HS}$ architectures is the woven asymmetry. The warp yarns and weft yarns are woven together with a certain regularity $[4,5]$. Since the float of fiber bundles is longer and the exchanges of the woven architecture are minimal, the 8HS architectures can be formed into complex shapes and still afford a certain degree of orthogonal reinforcement at the same time. However, because of the microstructural complexity and geometrical variables, modeling and analysis of the 8HS woven composites have always been challenging $[6,7]$. Firstly, complex microstructures are introduced into the composites by the interlacing fiber bundles, which must be modeled as realistically as possible in order to gather accurate predict results. Moreover, the composites involve a variety of material scales, including fiber/matrix scales, tow/coating scales, and microstructure scales, all of which should be considered accurately and appropriately [8-11].

Over the last few decades, several models have been developed to simulate the real structure and predict the effective mechanical properties of woven composites, including analytical and numerical models [12-16]. Rao et al. [17] characterized three different harness satin weave morphologies by the repeating unit cells, and introduced the concept of 'middle matrix layer'; Daggumati et al. [18] evaluated the local damage behavior by meso-scale FE simulation, and the results show that the stress and damage profiles are sensitive to the boundary condition and the unit model; Zhang and Hayhurst [19] proposed a finite-element-based approach for an 8HS woven ceramics matrix composite with and without tow waviness, and predicted the stressstrain and fracture behaviour by the unit cell model; Bassam et al. [20] also developed a multi-scale modeling approach for the 3D composites, which can capture the complex phenomena for different length scales, using the spatial Voronoi tessellation; Ullah et al. [21] presented a multiscale computational model to predict the nonlinear mechanical response of the fiber-reinforced polymer composites (FRP), using the periodic boundary conditions and uniform traction; Boise et al. [22] developed two multiphase voxel elements (MVEs) using standard finite elements for anisotropic composite materials to predict the effective stiffness tensor, and the results show that the new MVE have errors less than 1.5\%; Zhou et al. [23] established a porous model with multiscale of the silica/phenolic plain-woven composites, considering the effects of pores in the matrix, and deduced empirical formulas with effective thermal conductivity with temperature, fiber volume fraction, etc.; Unfortunately, few studies have been done on the multiscale model of composites, and the micromechanical modeling investigations of the $8 \mathrm{HS}$ woven composites are still insufficient at present. The accuracy of

\footnotetext{
* Corresponding author.

E-mail address: zmyang@imech.ac.cn (Z. Yang).
} 

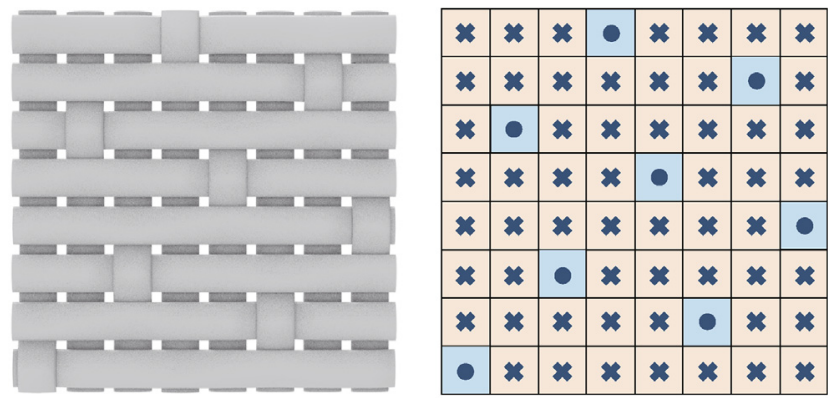

\section{* Warp Yarn up - Weft Yarn up}

Fig. 1. Schematic representation of orthogonal 8HS woven fabric.

modeling the $8 \mathrm{HS}$ woven composites for predicting mechanical properties is dependent on the material models, textile geometry, and modeling technique.

Recently, based on the framework of the finite element analysis (FEA) method, a number of investigators have developed the progressive damage modeling (PDM) technique for composites, which allows detailed strength and stress analysis and provides a better understanding of the failure behaviors of woven composites. Zhang et al. [11] developed a meso-scale model to simulate the failure behavior and local damage initiation for a triaxial braided composite, both the axial and transverse tension response were investigated; Cairns et al. [24] compared the discrete damage modeling (DDM) and continuum damage modeling (CDM) in the progressive damage analysis of wavy composite laminates, and the DDM shows better correlation with experimental results but CDM is more appropriate taking into account the nonlinear shear response; Wang et al. [25] investigated the damage initiation and failure behaviors of 2-D woven composites, and proposed an anisotropic damage model using fracture energy and the MurakamiOhno damage tensor; Shi et al. [26] modeled the failure properties of woven CMCs under tensile loading considering the inelastic deformation and the fiber orientations, based on the Tsai-Wu failure criterion; Yang et al. $[27,28]$ characterized the thermomechanical damage in oxide/oxide 8HS woven CMCs by thermal shock experiments, based on the continuum damage mechanics theory and thermodynamics law, and proposed a damage model for damage evolution in CMCs under cyclic thermal shock conditions. However, the failure process of $8 \mathrm{HS}$ woven composites considering the weave architecture is rarely reported, and the complex microstructure makes the prediction using numerical methods challenging.

The primary objective of this work is to develop a detailed multiscale modeling strategy that can reliably predict the failure behaviors of the $8 \mathrm{HS}$ woven composites. Firstly, two scales representative volume element(RVE) models are created and implemented by the finite element method (FEM), consisting of the fiber yarn modeling (micro-scale) and the woven fabric modeling (meso-scale). Then, the micromechanics failure criterions for matrix and fiber are introduced to gain a much deeper insight into the complex failure mechanisms of the 8HS woven composites under axis and transverse tensile loading. Furthermore, the tensile tests of the 8HS woven composites were conducted, and the failure mechanisms were observed and compared with the result of model predictions, which verify the correctness of the proposed multiscale models.

\section{Materials and experiments}

The 8HS woven composites investigated in this work are oxide/ oxide CMCs fabricated with uncoated Nextel ${ }^{\mathrm{TM}} 610\left(99 \% \alpha-\mathrm{Al}_{2} \mathrm{O}_{3}\right)$ fibers and a $\mathrm{Al}_{2} \mathrm{O}_{3}-\mathrm{SiO}_{2}-\mathrm{ZrO}_{2}$ matrix $\left(85 \mathrm{wt} \% \mathrm{Al}_{2} \mathrm{O}_{3}\right.$ and $15 \mathrm{wt} \%$ 3YSZ). The composites were processed by stacking 8 layers of $8 \mathrm{HS}$ woven fibers together, combining slurry infiltration and sintering at
Table 1

Mechanical properties of the components in oxide/oxide CMCs [29-31].

\begin{tabular}{llll}
\hline Parameter & Symbol & Value & Unit \\
\hline Elastic modulus of fiber & $E_{\mathrm{f}}$ & 379 & $\mathrm{GPa}$ \\
Elastic modulus of matrix & $E_{\mathrm{m}}$ & 210 & $\mathrm{GPa}$ \\
Density of fiber & $\rho_{\mathrm{f}}$ & 4.2 & $\mathrm{~g} / \mathrm{cm}^{3}$ \\
Density of matrix & $\rho_{\mathrm{m}}$ & 4.0 & $\mathrm{~g} / \mathrm{cm}^{3}$ \\
Volume fraction of fiber & $V_{\mathrm{f}}$ & 44 & $\%$ \\
Open porosity of composite $_{\text {Nextel }}^{\mathrm{TM}}$ 610 fiber diameter & $\zeta$ & 27.6 & $\%$ \\
Tensile strength of fiber $_{\text {Tensile strength of matrix }}$ & $\phi_{\mathrm{f}}$ & 10 & $\mu \mathrm{m}$ \\
\hline
\end{tabular}

$1000-1300{ }^{\circ} \mathrm{C}$. The finished composites panel is $2.8 \mathrm{~mm}$ in thickness, the density is $2.71 \mathrm{~g} / \mathrm{cm}^{3}$ with the fiber volume fraction of approximately $44 \%$. The properties of matrix and fiber are presented in Table 1. As mentioned in [32], the number and direction of fiber contained in the bundles may affect the modulus and strength of the bundles. However, the warp and weft fiber bundles are assumed to have the same material properties in the present work for the sake of simplicity.

The material properties and failure mechanisms of 8HS woven composites under tensile loading were evaluated by in-plane tensile testing. The specimens were cut along the fiber direction (the weft direction) from flat plates, and the dimensions are $160 \mathrm{~mm} \times 20 \mathrm{~mm} \times 2.8 \mathrm{~mm}$, which is shown in Fig. 2. To assure failure in the center regions and prevent failure in the clamping section, a reduced cross-section is produced for the specimens in the gauge areas. All the tests were performed under quasi-static loading using the servohydraulic MTS Bionix testing machine at room temperature in air, with a constant displacement rate of $0.006 \mathrm{~mm}^{\cdot \mathrm{min}^{-1}}$. The strains were measured with strain gauges. Besides, the stress-strain curves and the final failure modes of the specimens were recorded during the tests. The failure stress was measured from the maximum load.

\section{Modeling methodology}

The scanning electron microscope (SEM) observations in Fig. 3 have indicated that the microstructure of $8 \mathrm{HS}$ woven CMCs has a hierarchical architecture: (i) the woven composites composed of interlacing warp and weft yarns and $\mathrm{Al}_{2} \mathrm{O}_{3}-\mathrm{SiO}_{2}-\mathrm{ZrO}_{2}$ matrix surrounding (Fig. 3(a)); (ii) the fiber yarn (Fig. 3(b)), including individual fibers separated by $\mathrm{Al}_{2} \mathrm{O}_{3}-\mathrm{SiO}_{2}-\mathrm{ZrO}_{2}$ matrix. Therefore, a two-scale approach is established for the finite element modeling of the 8HS woven CMCs, which including the fiber yarn (micro-scale) modeling and the woven fabric (meso-scale) modeling. Fig. 4 illustrates the relevant characteristics of each scale considered in the multiscale analysis. The micro-scale model consists of fiber and $\mathrm{Al}_{2} \mathrm{O}_{3}-\mathrm{SiO}_{2}-\mathrm{ZrO}_{2}$ matrix. Considering that the voids between adjacent fibers are very small, they are ignored in the micro-scale model. The meso-scale model has two constituents including fiber yarns and $\mathrm{Al}_{2} \mathrm{O}_{3}-\mathrm{SiO}_{2}-\mathrm{ZrO}_{2}$ matrix.

\subsection{The micro-scale modeling}

The micro-scale model is concerned with the effective properties of fiber yarns, which can be regarded as a unidirectional composite composed of fibers and surrounding matrix (Fig. 5(a)).

For the fiber yarns, there are two types of representative volume element (RVE) shown in Fig. 5(a) which can be adopted to calculate the effective properties of fiber yarns: (i) The RVE including 4 semi-circles signed by the blue dash line; (ii) The RVE consists of 1 full circle and 4 quarter circles expressed by the red dash line. Besides, the simulate results of both RVE are completely consistent.

In the following study, the second type of RVE is created on microscale as demonstrated in Fig. 5(b). Definite $V_{F}^{\mathrm{f}}$ as the fiber volume 

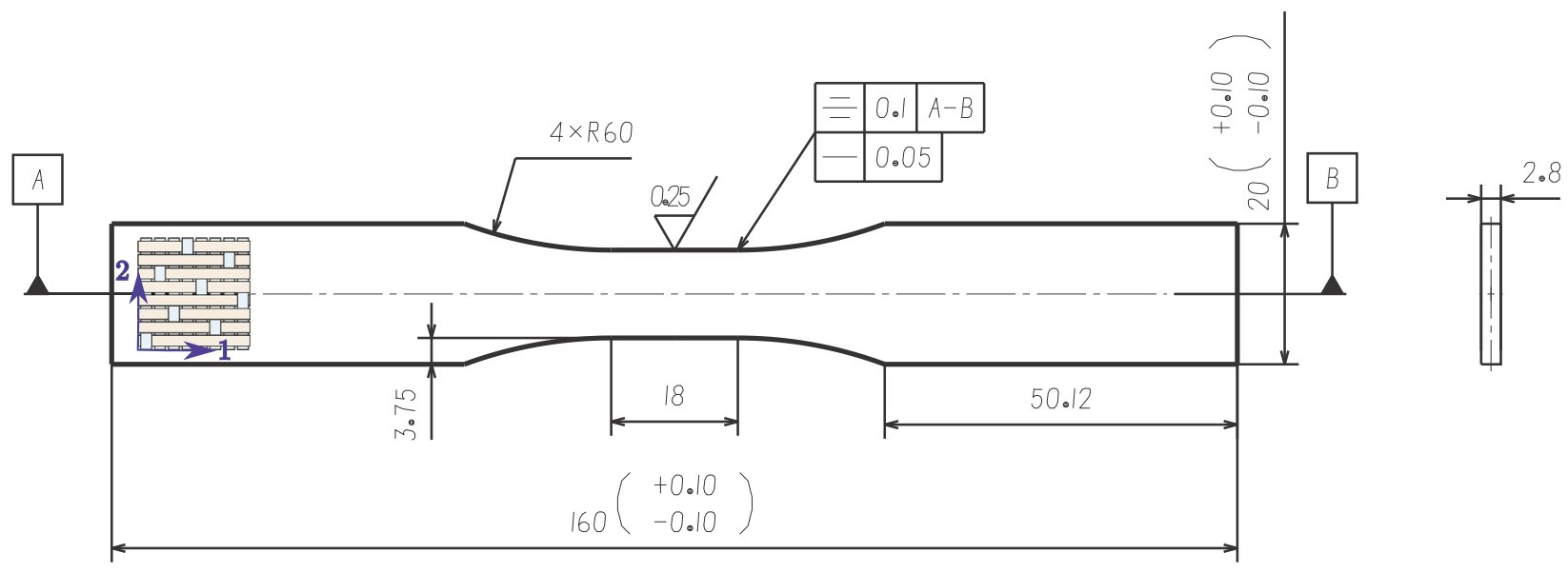

Fig. 2. Uniaxial tension specimen geometry of $8 \mathrm{HS}$ woven composites. All dimensions in mm.

fraction of the micro-scale model, then $V_{F}^{\mathrm{f}}$ can be expressed by the following:

$V_{\mathrm{F}}^{\mathrm{f}}=\frac{\pi \phi_{\mathrm{f}}^{2}}{2 L_{\mathrm{F}} W_{\mathrm{F}}}$

where $\phi_{\mathrm{f}}$ is the fiber diameter; $W_{\mathrm{F}}$ and $L_{\mathrm{F}}$ are width and length of the RVE, respectively. Then the fiber volume fraction of the micro-scale model is calculated to $65 \%$ in present work.

\subsection{The meso-scale modeling}

The meso-scale model is established to describe the stress-strain curve and failure mechanisms of 8HS woven composites under axial and transverse tensile loading. Based on the periodical characteristic of the 8HS woven composites, the meso-scale RVE is illustrated in Fig. 6(a), which is complex and difficult to analyse. It should be noted that besides the RVE, the unit cell (UC) can be also employed to estimate the properties of composites with smaller sizes and acceptable errors compared to the RVE, which is shown in Fig. 6(a) marked by the red dash line.

The geometric model of meso-scale UC containing fiber yarns and matrix is illustrated in Fig. 6(b). Different to the general plain weave, the meso-scale UC for 8HS weave is composed of four yarns: two are interlaced, the other two are parallel. Besides, a fully dense matrix is modeled to surround the yarns, and there is no gap between the adjacent yarns. The geometry parameters of the UC are obtained by the
SEM observation of the woven structure. The length and width of the UC are $L=882 \mu \mathrm{m}$, and the height of the ply is represented by $H=76 \mu \mathrm{m}$, as exhibited in Fig. 6(b).

Fig. 6(c) demonstrates the geometrical shape of a wavy yarn. The cross-section of the yarn is a lenticular shape consisting of two symmetrical arcs for simplicity, which has also been reported by many researchers $[33,34]$. The maximum thickness of the yarns is designed to $t$. Besides, the warp yarns are considered to be in complete contact with the weft yarns, then the centroidal path of the undulating yarns can be described by the sinusoidal waveform. Considering the local $x y z$ coordinate system of the UC shown in Fig. 6(c), the sinusoidal path can be obtained:

$h(x)=\sin \left(\frac{\pi x}{t}\right)$

where the local $x y z$ coordinate system is defined as that the $x$-direction is the sweep direction, and the $y z$-directions are in the cross-section plane.

\section{Development of finite element model}

\subsection{The failure criterion}

As discussed in Section 2, the material properties of matrix and fiber are different, and it should be noted that the tensile strength of matrix is far smaller than that of fiber, which will cause matrix failure precedes
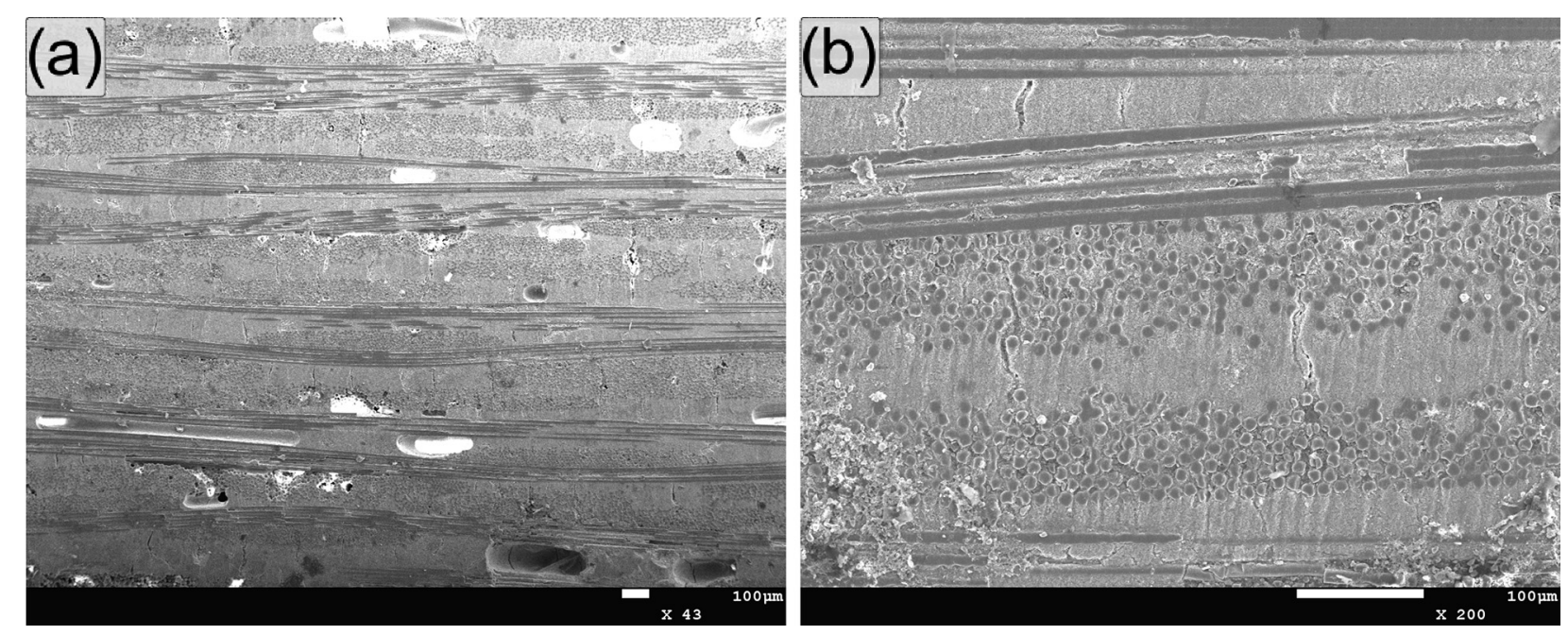

Fig. 3. SEM micrographs of the microstructure of the 8HS woven CMCs. (a) The cross-section of composites; (b) The fiber yarns. 


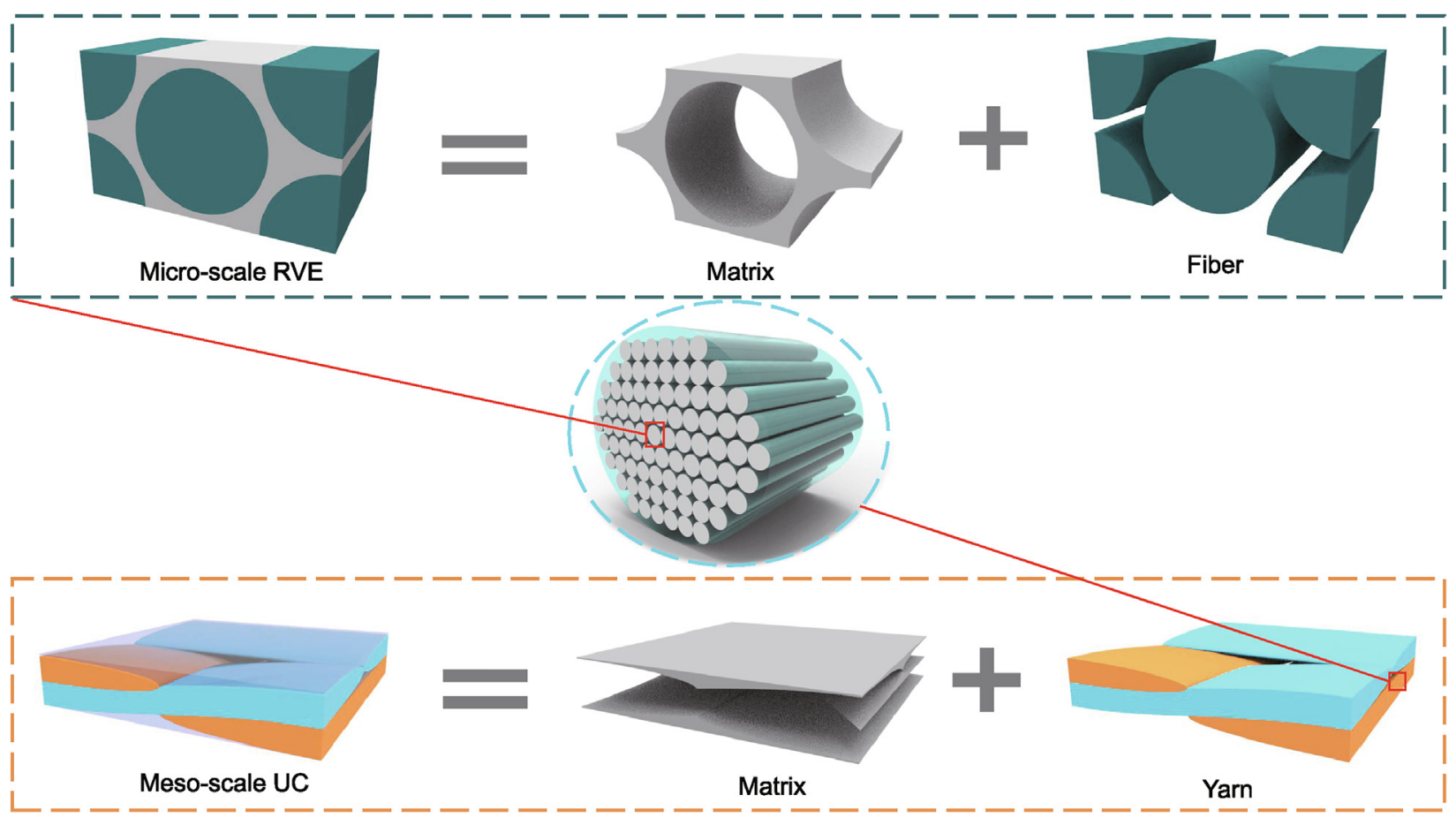

Fig. 4. The relevant characteristics of each scale of $8 \mathrm{HS}$ woven composites.

(a)
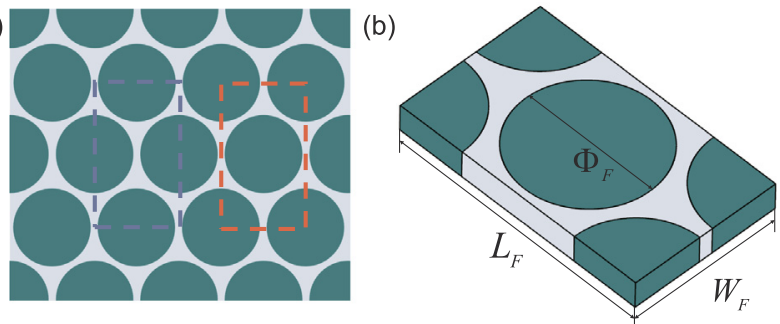

Fig. 5. Geometrical model of micro-scale RVE. (a) Different RVEs for a fiber yarn; (b) The micro-scale RVE.

fiber failure. Therefore, it is necessary to adopt different appropriate failure criteria for matrix and fiber to accurately investigate the failure mechanism of $8 \mathrm{HS}$ woven composites during tensile loading as well as to judge the failure initiation. The micromechanics of failure (MMF) criterion [35] was used to predict the failure mechanisms of the mesoscale model, and it uses the failure criterion of the fiber and matrix at the micro-level to determine the meso-level failure.

Due to the high longitudinal strength and stiffness of fibers, the
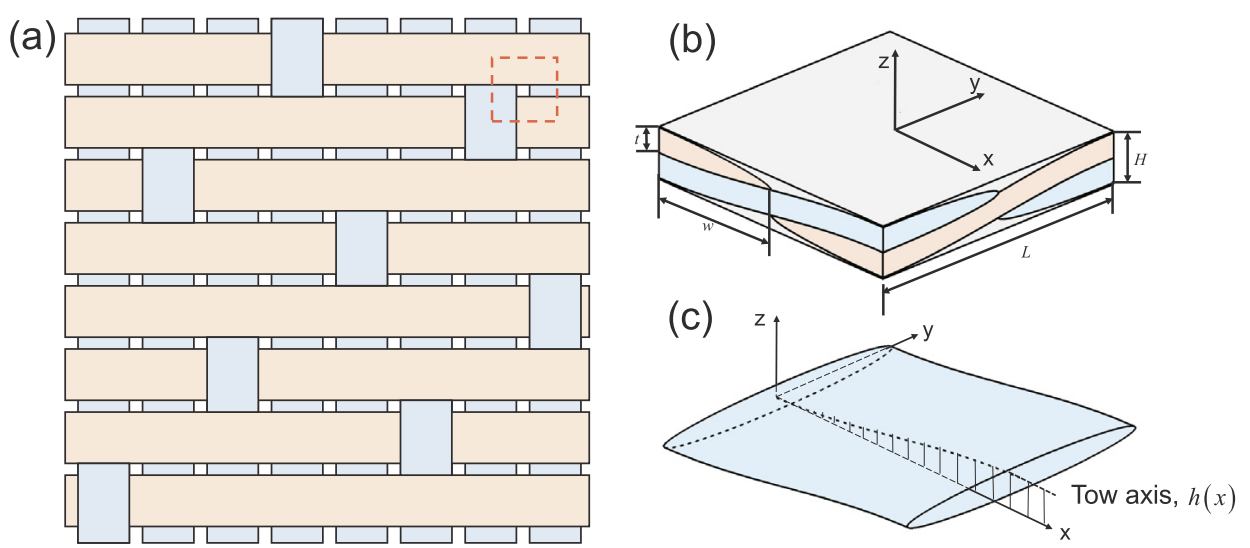

Fig. 6. Geometrical model of meso-scale. (a) The RVE model, (b) the UC model, (c) the geometrical shape of a wavy yarn. 


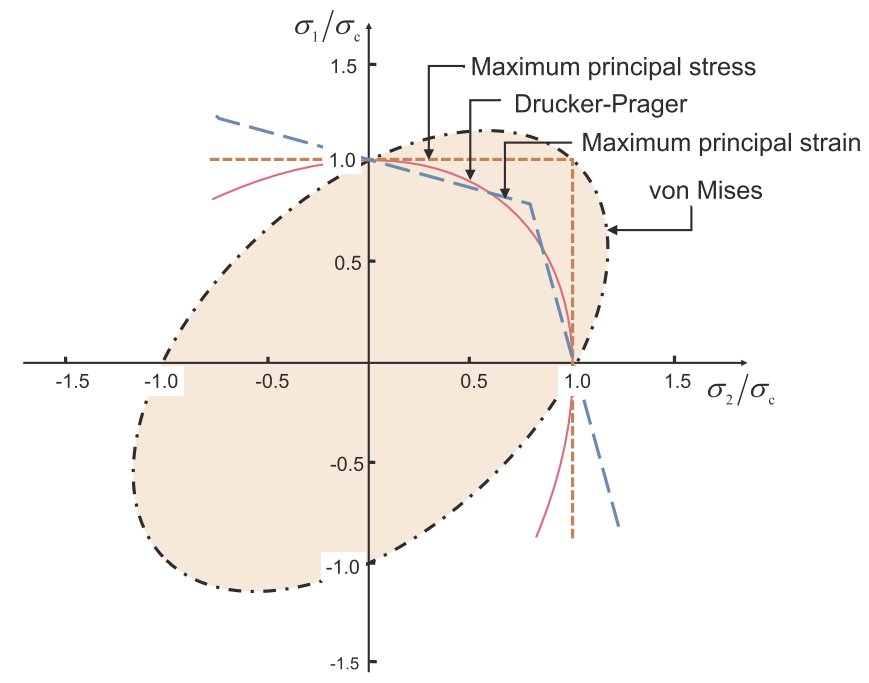

Fig. 7. Schematic of the failure surface defined by the linear version of the maximum principal stress.

criterion for matrix is formulated:

$\frac{\left(\sigma_{\mathrm{vm}}^{\mathrm{m}}\right)^{2}}{T_{\mathrm{m}}}+\frac{J_{1}^{\mathrm{m}}}{T_{\mathrm{m}}}=1$

where $T_{\mathrm{m}}$ is the matrix tensile strength; $J_{1}^{\mathrm{m}}$ is the first stress invariant and $\sigma_{\mathrm{vm}}^{\mathrm{m}}$ is the von Mises stress of the matrix. It should be noted that Eq. (5) can also be employed in judging the failure of the matrix in the meso-scale model, then $J_{1}^{\mathrm{m}}$ and $\sigma_{\mathrm{vm}}^{\mathrm{m}}$ turn into the meso-scale stress components.

\subsection{Material damage constitutive models}

The matrix is initially isotropic, with elastic modulus $E_{m}$ and poisson ratio $\nu_{\mathrm{m}}$. Therefore, the elastic behavior of matrix can be characterized using the free enthalpy potential as following [36]:

$2 \rho^{\mathrm{m}} \phi_{0}^{\mathrm{m}}(\underline{\underline{\sigma}})=\underline{\underline{\sigma}}: \underline{\underline{\underline{E}}}_{0}^{\mathrm{m}-1}: \underline{\underline{\sigma}}$

where $\rho^{\mathrm{m}}$ is the density of matrix, $\phi_{0}^{\mathrm{m}}$ is the free enthalpy potential. The elastic state law is written as:

$\underline{\underline{\varepsilon}}^{\mathrm{m}}(\underline{\underline{\sigma}})=\frac{\partial \rho^{\mathrm{m}} \phi_{0}^{\mathrm{m}}}{\partial \underline{\underline{\sigma}}}(\underline{\underline{\sigma}})=\underline{\underline{\underline{E}}}_{0}^{\mathrm{m}}: \underline{\underline{\sigma}}$

where $E_{0}^{\mathrm{m}}$ is the initial elastic modulus of matrix. The elastic behavior is depended on two independent material constants $E_{\mathrm{m}}, \nu_{\mathrm{m}}$.

As for the matrix-impregnated warp and weft yarns, they are assumed to transversely isotropic materials, which need five independent material constants to obtain the elastic response of materials. The FE analysis of the micro-scale RVE or the Chamiss equations can be used to obtain the material constants of the fiber yarns [37,38]. In this work, the FE analysis was used to calculate these material parameters, which can provide more reasonable values, and the Chamiss equations are employed to further verify the numerical results, which are defined as:

$\left\{\begin{array}{l}E_{1}^{\mathrm{y}}=E^{\mathrm{f}} V^{\mathrm{f}}+E^{\mathrm{m}} V^{\mathrm{m}} \\ \frac{1}{E_{2}^{\mathrm{y}}}=\frac{1}{E_{3}^{\mathrm{y}}}=\frac{V^{\mathrm{f}}}{E^{\mathrm{f}}}+\frac{V^{\mathrm{m}}}{E^{\mathrm{m}}} \\ \frac{1}{v_{12}^{\mathrm{y}}}=\frac{1}{v_{13}^{\mathrm{y}}}=\frac{V^{\mathrm{f}}}{v^{\mathrm{f}}}+\frac{V^{\mathrm{m}}}{v^{\mathrm{m}}} \\ \nu_{23}^{\mathrm{y}}=v^{\mathrm{f}} V^{\mathrm{f}}+v^{\mathrm{m}} V^{\mathrm{m}} \\ \frac{1}{G_{12}^{\mathrm{y}}}=\frac{1}{G_{13}^{\mathrm{y}}}=\frac{V^{\mathrm{f}}}{G^{\mathrm{f}}}+\frac{V^{\mathrm{m}}}{G^{\mathrm{m}}} \\ G_{23}^{\mathrm{y}}=G^{\mathrm{f}} V^{\mathrm{f}}+G^{\mathrm{m}} V^{\mathrm{m}}\end{array}\right.$

where $V^{\mathrm{f} / \mathrm{m}}, E^{\mathrm{f} / \mathrm{m}}, v^{\mathrm{f} / \mathrm{m}}$ and $G^{\mathrm{f} / \mathrm{m}}$ are the volume fraction, elastic modulus, poisson ratio and shear modulus of the initial fiber/matrix, respectively.

After satisfying the damage initiation criterion, the degradation of material stiffness will occur, which is caused by further loading, and it is controlled by damage variables. The range of damage variables is between 0 (undamaged state) and 1 (fully damaged state). Thus, the free enthalpy potential of the damaged materials can be rewritten as:

$2 \rho \phi(\underline{\underline{\underline{\sigma}}}, \underline{\underline{\underline{\underline{D}}}})=\underline{\underline{\underline{\sigma}}}: \underline{\underline{\underline{\underline{D}}}}: \underline{\underline{\sigma}}$

where $\underline{\underline{\underline{\underline{D}}}}$ is the damage variable. For the initial materials, it has $\underline{\underline{\underline{\underline{D}}}}_{0}={\underline{\underline{\underline{\underline{E_{0}}}}}}^{-1}$. Then the damage variable can be written as:

$\underline{\underline{\underline{\underline{D}}}}=\frac{1}{S}\left[\begin{array}{ccc}\left(1-s_{\mathrm{f}}\right) E_{1} & \omega \nu_{21} E_{1} & 0 \\ \omega \nu_{12} E_{2} & \left(1-s_{\mathrm{m}}\right) E_{2} & 0 \\ 0 & 0 & \left(1-s_{\mathrm{s}}\right) G_{12}\end{array}\right]$

where

$\omega=\left(1-s_{\mathrm{f}}\right)\left(1-s_{\mathrm{m}}\right)$

and $S=1-\omega \nu_{12} \nu_{21} ; s_{\mathrm{m}}, s_{\mathrm{f}}$ and $s_{\mathrm{s}}$ are the internal damage variables of matrix, fiber and shear damage, respectively, and they have the form as follows:

$s_{\mathrm{f}}= \begin{cases}s_{\mathrm{ft}} & \sigma_{11}>0 \\ s_{\mathrm{fc}} & \sigma_{11}<0\end{cases}$

$s_{\mathrm{m}}= \begin{cases}s_{\mathrm{mt}} & \sigma_{22}>0 \\ s_{\mathrm{mc}} & \sigma_{22}<0\end{cases}$

$s_{\mathrm{s}}=1-\left(1-s_{\mathrm{ft}}\right)\left(1-s_{\mathrm{fc}}\right)\left(1-s_{\mathrm{mt}}\right)\left(1-s_{m c}\right)$

where the subscripts of $t, c$ means the tensile or compress loading.

As the evolution of damage variable is controlled by equivalent displacements, for a particular mode, the damage variable $s_{I}(I=\mathrm{ft}, \mathrm{fc}, \mathrm{mt}, \mathrm{mc})$ can be defined by:

$S_{I}=\frac{\delta_{I, e q}^{\mathrm{f}}\left(\delta_{I, e q}-\delta_{I, e q}^{0}\right)}{\delta_{I, e q}\left(\delta_{I, e q}^{\mathrm{f}}-\delta_{I, e q}^{0}\right)}$

where $\delta_{I, e q}^{0}$ is the initial equivalent displacement satisfying the damage initiation criterion in the particular damage mode; $\delta_{I, e q}$ is the equivalent displacement, and $\delta_{I, e q}^{\mathrm{f}}$ is the equivalent displacement of the completely damaged material $\left(s_{I}=1\right)$ and can be computed from the equivalent stress and fracture energy at which the initiation criterion is met. The equivalent displacement of the failure mode is represented by the corresponding components of the effective stress components used in the failure criteria.

Then, the state law of the damaged materials can be simply derived from Eq. (9):

$\underline{\underline{\varepsilon}}(\underline{\underline{\sigma}}, \underline{\underline{\underline{\underline{D}}}})=\frac{\partial \rho \phi}{\partial \underline{\underline{\sigma}}}(\underline{\underline{\sigma}}, \underline{\underline{\underline{\underline{D}}}})=\underline{\underline{\underline{\underline{D}}}}: \underline{\underline{\sigma}}$

\subsection{Periodic boundary conditions}

As the meso-scale UC model has been established, the periodic boundary conditions (PBCs) need to be employed to all surfaces of the model, in which case the entire composite can be represented by the meso-scale UC model. The PBCs use translational symmetry transformations to effectively handle the displacements, strains and stresses related to the UC model, which involve transferring the stress and strain of a particular UC to any other UC by the identical transformation. Due to the similar nature of the displacements and strains, both of them can be transformed as each other.

The geometric model of meso-scale UC is a regular cuboid, then the PBCs can be defined as: 
$u_{i}^{+}=u_{i}^{*}+\bar{\varepsilon}_{i k} x_{k}^{+}$

$u_{i}^{-}=u_{i}^{*}+\bar{\varepsilon}_{i k} x_{k}^{-}$

where $u_{i}^{+}$and $u_{i}^{-}$are the displacement components of each pair of the relative surfaces, $u_{i}^{*}$ is the periodic part of displacement components; $\bar{\varepsilon}_{i k}$ is the average strain of UC; $x_{k}^{+}$and $x_{k}^{-}$are the position coordinate of the UC model. Then the relative displacement $\Delta x_{k}$ of the two relative surfaces can be defined using Eq. (16):

$u_{i}^{+}-u_{i}^{-}=\left(x_{k}^{+}-x_{k}^{-}\right) \bar{\varepsilon}_{i k}=\Delta x_{k} \bar{\varepsilon}_{i k}$

Considering that the value of $\Delta x_{k}$ is constant for $\mathrm{x}, \mathrm{y}$ and $\mathrm{z}$ direction for each increment step, Eq. (17) becomes:

$u_{i}^{+}-u_{i}^{-}=c_{i}$

where $c_{i}$ is a constant. In this study, assume that the displacement components of the node in the three relative faces are equal at each step, and the surfaces $x^{-}, y^{-}$and $z^{-}$are the symmetric faces. By setting the linear displacement constraint between each pair of nodes on the parallel boundary surfaces, Eq. (18) can be easily implemented.

Volume average stresses and strains can be obtained by:

$\bar{\sigma}_{i j}=\frac{1}{V} \int_{V} \sigma_{i j} d V=\frac{1}{V} \sum_{k=1}^{n} \sigma_{i j}^{k} V^{k}$

$\bar{\varepsilon}_{i j}=\frac{1}{V} \int_{V} \varepsilon_{i j} d V=\frac{1}{V} \sum_{k=1}^{n} \varepsilon_{i j}^{k} V^{k}$

where $V$ represents volume of the UC; $k$ represents integration points in the UC; $V^{k}, \sigma_{i j}^{k}, \varepsilon_{i j}^{k}$ are volume, stress and strain associated with $k$ th integration point.

\subsection{Mechanical property of micro-scale model}

The key to accurately simulate the failure mechanism of meso-scale model is obtaining the effective properties of fiber yarns. Since the fiber yarns consist of matrix and fibers, they are assumed to be transversely isotropic, and the material property is different from each component. Therefore, the micro-scale model has been generated to predict the axial and transverse effective properties of fiber bundles, which will be then used in the meso-scale numerical model.

The micro-scale FE model is established in ABAQUS on the basis of the component properties listed in Table 1 and the above PBCs. Fig. 8 illustrates the stress distributions of micro-scale RVE in the axial (xaxis) direction tensile loading and transverse (y-axis) direction tensile loading. The stress field of the micro-scale RVE model is harmonious under tensile loading. When subjected to the longitudinal tensile load, the fiber plays a major role in loading, and the matrix acts as a supporting, which is determined by the transverse homogeneity of the unidirectional fiber-reinforced composites. Under the action of
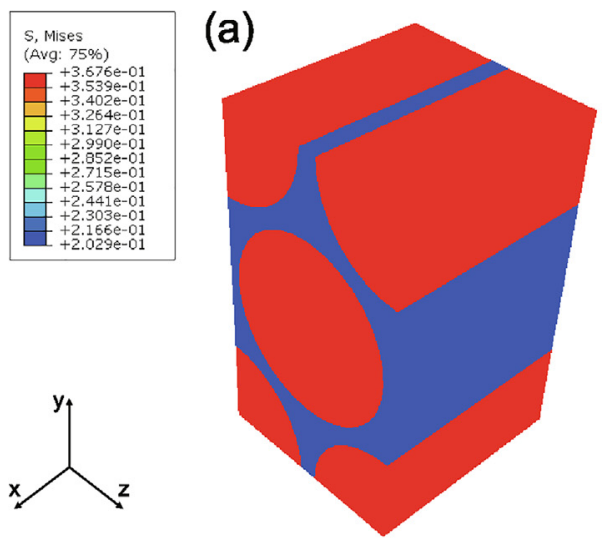

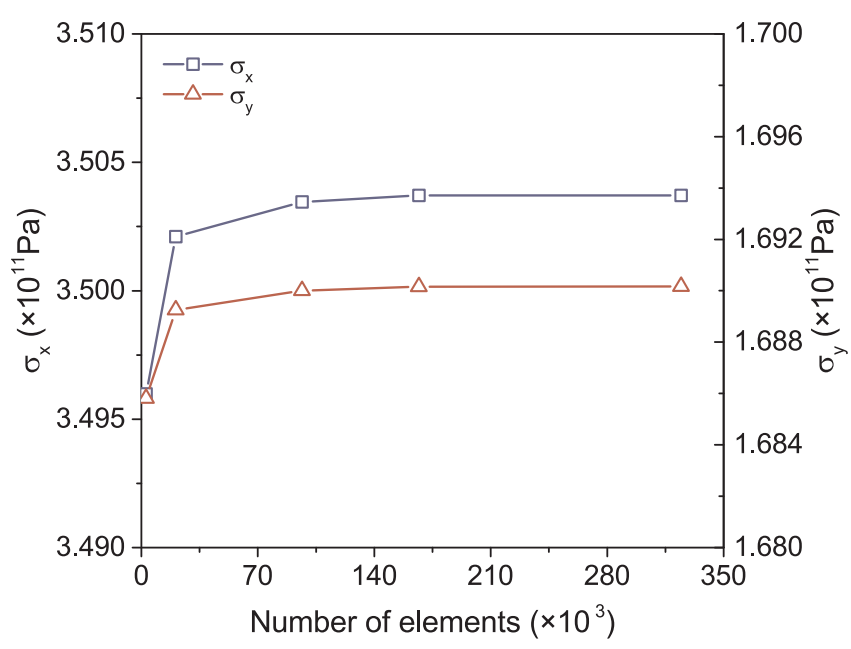

Fig. 9. Mesh independence for micro-scale RVE.

transverse tensile load, the matrix plays a major bearing force, and the variation of the displacement field along the lateral direction shows non-uniformity, which is caused by the non-uniformity of the transverse elastic modulus of the RVE model.

Moreover, in order to ensure the validity and accuracy of the computed effective properties, multiple analyses on models with different mesh densities are carried out to check the convergence of the results. The parameters of interest $\sigma_{x}$ and $\sigma_{y}$ are selected to perform the mesh independence studies, which can numerically approximate the corresponding elastic modulus $E_{x}$ and $E_{y}$. Fig. 9 shows the mesh independence of $\sigma_{x}$ and $\sigma_{y}$ with increasing number of elements, it clearly illustrates that the results are convergent when the number of element mesh is 192,000, ensuring the correctness of results. Therefore the effective properties of fiber bundles are obtained.

\section{Results and discussions}

After establishing the meso-scale UC model of the 8HS woven composites and obtaining the effective properties of each component in the meso-scale model, the analysis of the meso-scale model under axial and transverse tensile loading was conducted by ABAQUS/Standard. The meso-scale model was meshed using 64676 eight-noded finite elements and the node number is 83739 . Besides, the fiber bundles and the matrix were assembled as a whole model when importing ABAQUS to ensure the coordinating deformation between the interactions of fibers and matrix. The fiber bundles were treated as transversely isotropic materials and the matrix is isotropic, which prosperities have

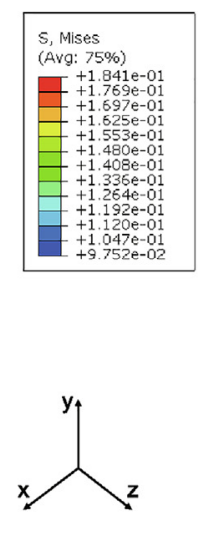

Fig. 8. Stress distributions of micro-scale RVE in the (a) $x$ direction, (b) $y$ direction. 


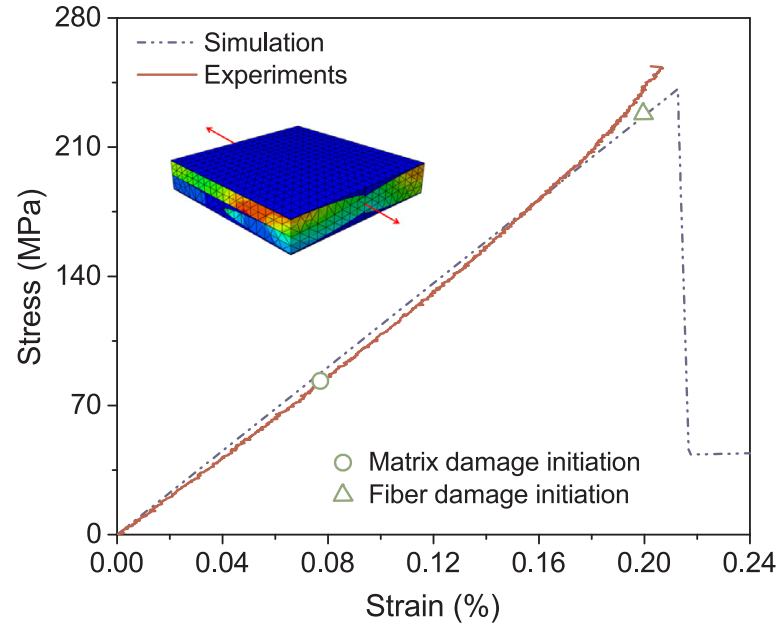

Fig. 10. Stress-strain curve of prediction and experimental results in the axis direction.

been described earlier.

The experimental results are further used to compare with the global stress-strain responses to verify the accuracy of the proposed model. Besides, the axial and transverse tensile failure processes of the 8HS woven composite are discussed in detail.

\subsection{Global stress-strain responses}

The meso-scale model is subjected to uniaxial tensile load in the warp direction (z-direction) and the transverse direction( $x$-direction). The macroscopic effective stress for the loading section is defined by computing the reaction forces on the loading surface, and divided by the area of the loading surface, while the effective strain is determined by the total elongation dividing the total length in the loading direction of the model. Then the global stress-strain responses can be obtained.

Fig. 10 shows the predicted macroscopic stress-strain curve in the warp direction plotted together with the experimental one. Clearly, the simulated stress-strain response is in good agreement with the experimental result, which verifies the effectiveness of the proposed multiscale modeling strategy. Furthermore, the computed curve is approximately linear, while the experimental curve is nonlinear to some extent, so that the initial modulus of simulation is a little higher than the experimental modulus, and the predicted ultimate strength is slightly lower than the experimental ultimate strength. The reasons are discussed below: As CMCs are porous matrix materials, the stiffness of porous matrix is lower than the pure matrix listed above, resulting in the lower modulus in the beginning. After the matrix failure, the fibers begin to bear the load, and due to the higher strength of fibers, the ultimate strength of the material is slightly raised.

Moreover, the damage in matrix initiates at $\varepsilon=0.08 \%$, but the stressstrain curve has no significant change, which reveals that the matrix damage has little effects on the elastic behavior of the woven composites under axial tensile load. When the global strain level is $0.2 \%$, the axial fiber bundles will undergo fracture and destruction, and finally, result in the ultimate failure of the material when the strain level is $0.22 \%$.

The predicted stress-strain curve in the transverse direction is shown in Fig. 11. The stress-strain curve is approximately linear until the global strain reaches $0.6 \%$, which indicates the tensile matrix failure initiation. Besides, there is nearly no fiber damage during the specified loading range, which may due to that after the matrix failure, the model loses the load-carrying capacity because the loading is directly applied to the matrix. Moreover, the material under axial tensile loading behaves stiffer compared to that under transverse loading due to the same reason mentioned above.

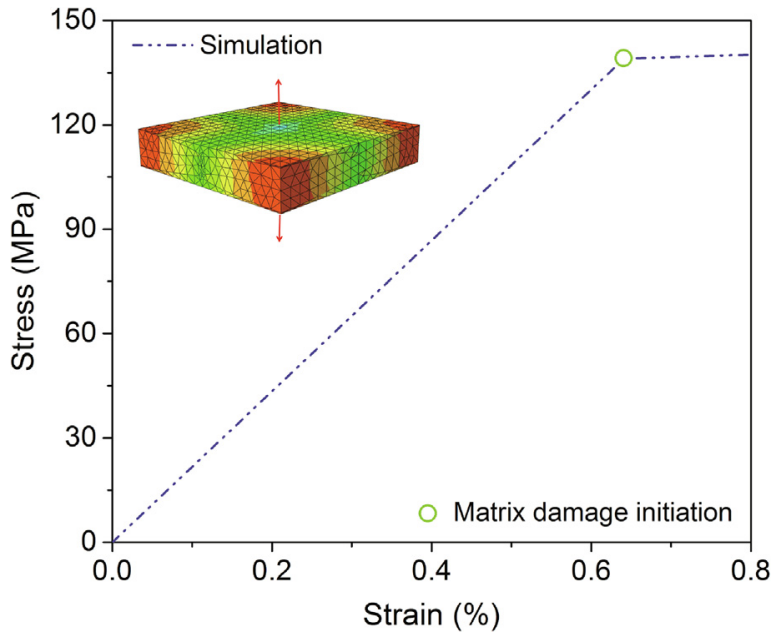

Fig. 11. Stress-strain curve of predict results in the transverse direction.

The stress distributions of the matrix, warp yarn and weft yarn in the meso-scale UC before the catastrophic failure under axial tensile loading is displayed in Fig. 12(a). The stress distribution of different components has its characteristics based on the location, property and weave structure. The stress level of warp yarn is much higher than that of weft yarn and matrix, which indicates that the main load of warp yarn is longitudinal tension. Due to the periodical contact of the fiber yarns at the crimp zones, the significant bending-torsion effects are produced even under simple uniaxial loading. In addition, the interaction between the warp and weft yarns is complex, resulting in the obvious stress concentration in the yarn crimp regions, which indicates that the matrix damage and fiber fracture will initiate at these locations ideally.

Compared to the stress distribution in the axis direction, the stress distribution in the transverse direction is more uniform as shown in Fig. 12(b). As the loading is along the $\mathrm{x}$-direction, all the components are uniformly stretched. The locations of the highest stresses are the corners of the UC, and the center of the UC shows the lowest stress, which may due to the center is composed of the matrix, and the matrix has the lower elastic modulus. The stress in the matrix and fiber is the same order of magnitude, which indicates that the matrix will break first since the strength of the matrix is lower than fiber. The stress distributions exhibit an intuitive view of the initial position of damage in the matrix and fiber, and the effect of different fiber yarns under the axial and transverse tensile loadings.

\subsection{Failure process under axial tensile loading}

After obtaining the stress distributions under axial tension loading, which have exhibited the effects of the weave architecture within the UC, the failure mechanism of the meso-scale UC is investigated at average strain levels of $0.1 \%, 0.2 \%$ and $0.22 \%$ by carrying out the FE analysis and the failure criterion, as displayed in Fig. 13.

For the global strain level of $0.1 \%$, the matrix damage has been initiated, and there are some elements of matrix failure in the loading direction, and the yarns support higher stress levels without damage. With strain increases, however, the matrix damage will accumulate to the saturation state before fiber failure, the UC still displays a good load-bearing capacity as displayed in the stress-strain curves. When the global strain level reaches $0.2 \%$, yarn failure was first observed in the crimp zones of warp yarns, due to the stress concentration and highstress level. At the same time, the matrix has lost its carrying capacity. Then the damage propagates in the transverse direction perpendicular to the loading direction, and the weft yarns failure. Finally, at $\varepsilon=0.22 \%$, the catastrophic failure occurs in the whole woven structure, the sudden fall of the stress-strain curve also proved this phenomenon. 

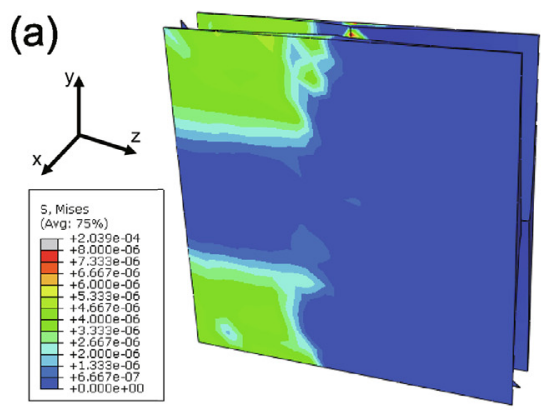

Matrix

(b)

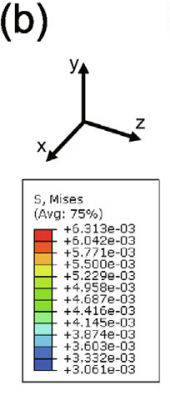

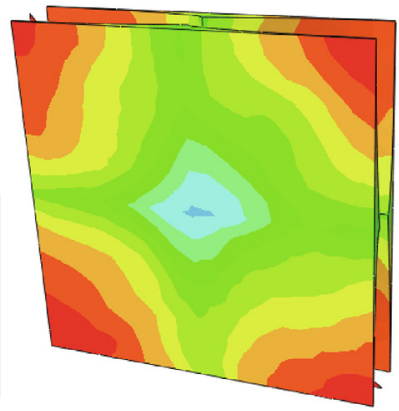

Matrix
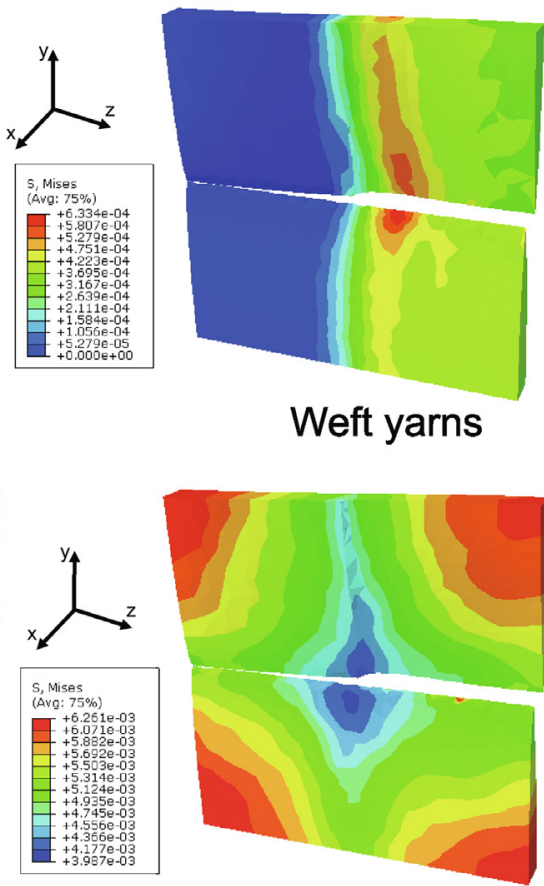

Weft yarns

Weft yarns
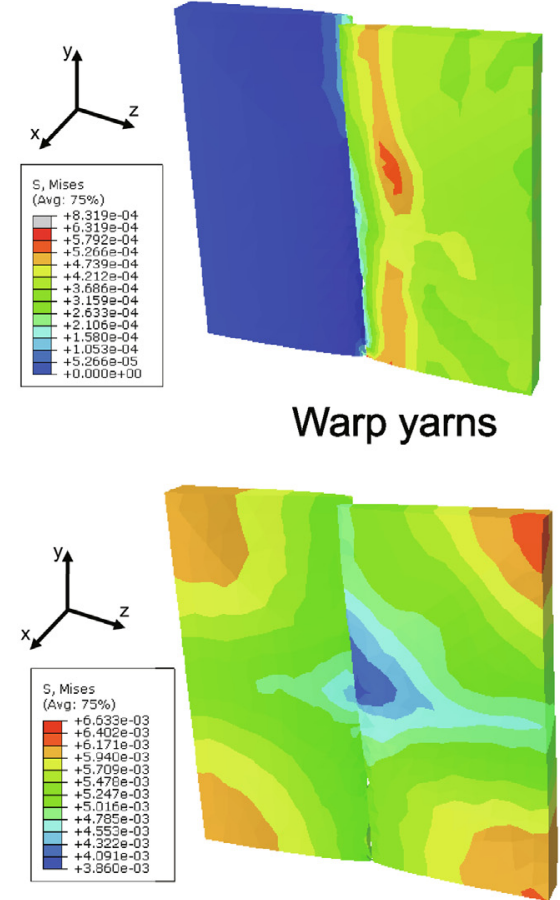

Warp yarns

Fig. 12. Stress distributions (unit: $\times 10^{11} \mathrm{~Pa}$ ) under tensile loading: (a) in the axis direction; (b) in the transverse direction.

Fig. 13(c) shows the final rupture location of the UC at $0.22 \%$, the UC model was pulled from the middle section perpendicular to the loading direction.

Furthermore, the predicted failure behaviors can be compared with the experimental observations using the SEM. Fig. 14 presents the final failure state the test specimens under axis tensile loading. Both matrix cracking and fiber yarn fracture can be clearly identified, which agree well with the predicted failure behaviors, indicating that the final failure mechanism is fiber fracture and pull-out.

It can conclude that the failure mechanism of the 8HS woven composite under axis tensile loading is the matrix failure occurs at the first stage which will not affect the material carrying capacity, and the fiber failure leads to the final failure of the total material. Besides, the fiber failure exhibits more obvious brittle behavior when comparing to the matrix failure, and the stress concentration in the crimp zones lower the ultimate strength of the model and induce the early damage initiations at the crimp zones. By comparing the results with the stress distribution and experimental observation, the proposed meso-scale model can precisely predict the final rupture locations and the different failure patterns in the $8 \mathrm{HS}$ woven composites.

\subsection{Failure process under transverse tensile loading}

Similar to the axis tension loading case, Fig. 15 shows the predicted failure mechanism of meso-scale UC model at global strain levels of

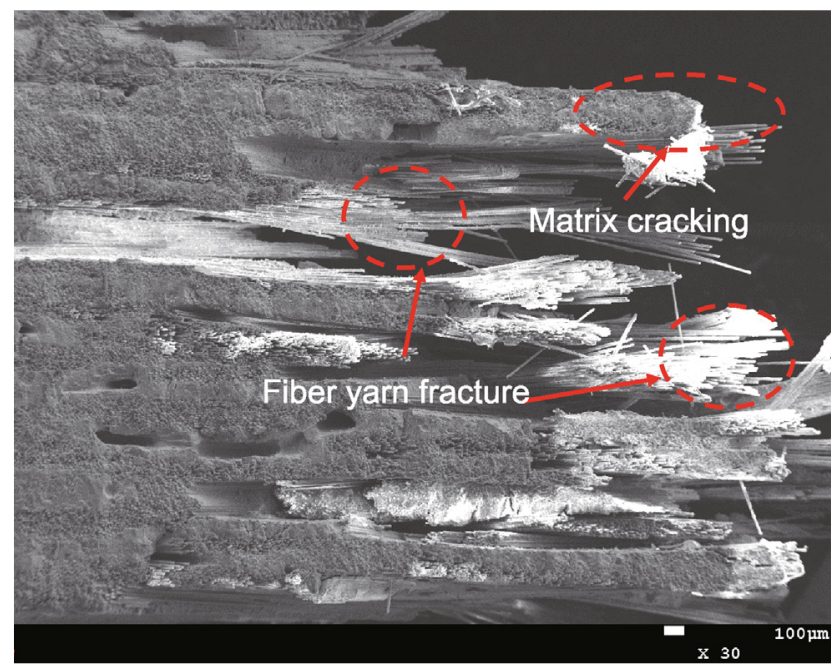

Fig. 14. SEM micrograph of the final failure zone.

$0.6 \%, 0.7 \%$ and $0.8 \%$ under transverse tensile loading. When the global strain $\varepsilon$ reaches $0.6 \%$, the matrix begins to fail, and it spreads very quickly across the loading plane, which may results in delamination in the composites. However, with strain increases, there are no obvious
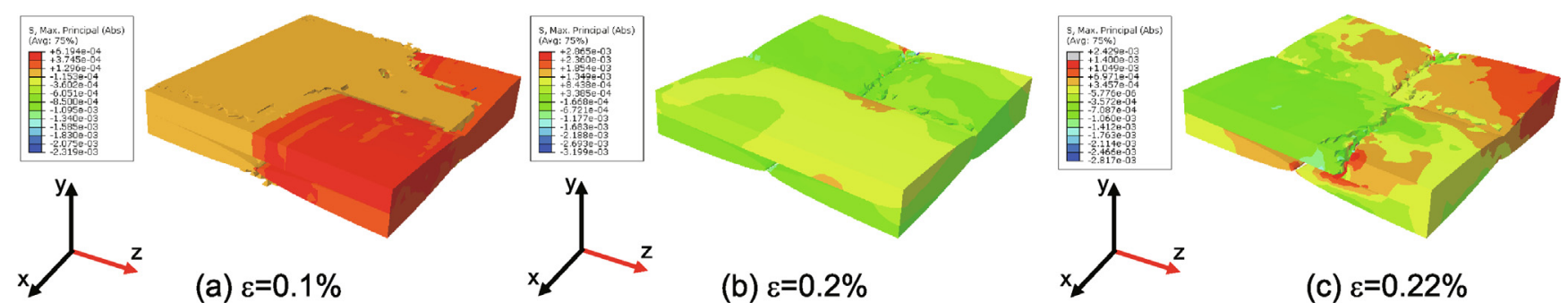

Fig. 13. Failure process with the increase strain under axis tensile loading.

(c) $\varepsilon=0.22 \%$ 


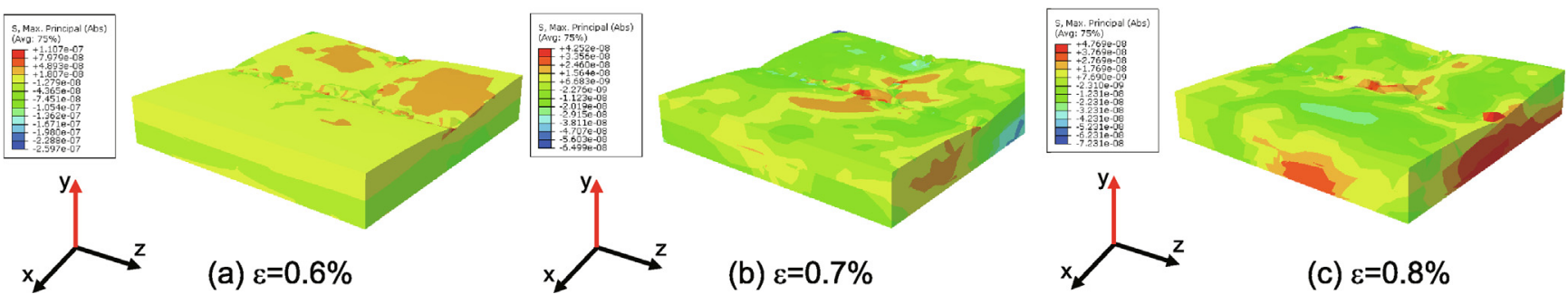

Fig. 15. Failure process with the increase strain under transverse tensile loading.

cracks existing in fiber, due to that the UC loses its bearing capacity and little loading transmitted to the fiber, so that the failure strength of the fiber is not reached. The failure mechanisms of the woven composites under transverse tensile loading are delamination and matrix cracking. Due to the limitation of experimental conditions, no transverse tensile test was conducted.

The different failure mechanisms between the axis and transverse tensile loading may due to that: When under the axis tensile loading, the fiber supports almost the entire load applied, because of the higher strength and modulus compared to the matrix and the fiber is longitudinally continuous. Similarly, in the transverse loading case, matrix plays a similar role as fiber does under axis load, so that the matrix strength is the major factor to determine the failure mechanisms under this circumstance.

\section{Conclusions}

In this work, multiscale models were established to study the failure mechanism for 8HS woven composites under axis and transverse tensile loading. The global stress-strain response of the 8HS woven oxide/oxide CMCs under axial and transverse tension loading was simulated and compared with the experimental results, which correlated reasonably well. Moreover, the failure mechanisms of the woven composites were investigated by the multiscale models. Following conclusions can be drawn from the work:

- The multiscale models of the 8HS woven composites are developed consisting of fiber yarn (micro-scale) model and the woven fabric (meso-scale) model. The micro-scale RVE model was generated to predict the effective properties of the transversely isotropic fiber bundles and the meso-scale UC model instead of the RVE was established to simulate the stress-strain response and failure mechanism under tensile loading. Both of them are based on the practical geometric dimension and material properties.

- The micromechanics of failure criterion was used for the different appropriate failure criteria of matrix and fiber, which has been reflected in the stress-strain curves. The predicted modulus and ultimate strength from the stress-strain curves show a good agreement with the experimental results.

- The crimp zones of the 8HS woven composites are the weakness places due to the obvious stress concentrations, which have been verified by the simulated stress distributions of the meso-scale model. The stress concentration finally leads to fiber fracture and matrix cracking.

- The failure mechanisms of the 8HS woven CMCs under axis tensile loading are the matrix cracking and fiber fracture, and the ultimate catastrophic failure is caused by the failure of fiber yarns. For the transverse loading case, the failure mechanisms consist of delamination and matrix cracking.

This framework provides a significant insight into understanding how the 8HS weave architecture contributes to the global stress response and failure mechanisms of the materials. Besides, the proposed multiscale models can be extended to other textile composites.

\section{CRediT authorship contribution statement}

Zhengmao Yang: Writing - original draft, Writing - review \& editing, Data curation, Methodology, Conceptualization. Han Yan: Investigation, Validation, Software.

\section{Declaration of Competing Interest}

The authors declare that they have no known competing financial interests or personal relationships that could have appeared to influence the work reported in this paper.

\section{Acknowledgements}

The present work is supported by the Strategic Priority Research Program of Chinese Academy of Sciences (Grant No. XDA17030100). The authors would like to thank the reviewers for their fruitful discussions and constructive comments.

\section{References}

[1] Rajan VP, Zok FW. Effects of non-uniform strains on tensile fracture of fiber-reinforced ceramic composites. J Mech Phys Solids 2012;60(12):2003-18.

[2] Döbrich Oliver, Gereke Thomas, Cherif Chokri. Modeling the mechanical properties of textile-reinforced composites with a near micro-scale approach. Compos Struct 2016;135:1-7.

[3] Elias A, Laurin F, Kaminski M, Gornet L. Experimental and numerical investigations of low energy/velocity impact damage generated in 3D woven composite with polymer matrix. Compos Struct 2017;159:228-39.

[4] Searles K, Odegard G, Kumosa M. Micro- and mesomechanics of 8-harness satin woven fabric composites: I-evaluation of elastic behavior. Compos Part A 2001;32(11):1627-55.

[5] Chateau C, Gelebart L, Bornert M, Crepin J, Caldemaison D, Sauder C. Modeling of damage in unidirectional ceramic matrix composites and multi-scale experimental validation on third generation SiC/SiC minicomposites. J Mech Phys Solids 2014;63:298-319.

[6] Ayranci Cagri, Carey Jason. 2D braided composites: a review for stiffness critical applications. Compos Struct 2008;85(1):43-58.

[7] Yang Zhengmao, Liu Hui. Effects of thermal aging on the cyclic thermal shock behavior of oxide/oxide ceramic matrix composites. Mater Sci Eng: A 2020;769:138494https://doi.org/10.1016/j.msea.2019.138494.

[8] Landis Chad M, Beyerlein Irene J, McMeeking Robert M. Micromechanical simulation of the failure of fiber reinforced composites. J Mech Phys Solids 2000;48(3):621-48.

[9] Borkowski Luke, Chattopadhyay Aditi. Multiscale model of woven ceramic matrix composites considering manufacturing induced damage. Compos Struct 2015;126:62-71.

[10] Dai S, Cunningham PR. Multi-scale damage modelling of 3D woven composites under uni-axial tension. Compos Struct 2016;142:298-312.

[11] Zhang Dianyun, et al. A micromechanics based multiscale model for nonlinear composites. Acta Mech 2014;225:1391-417.

[12] Tan P, Tong L, Steven GP. Modelling for predicting the mechanical properties of textile compositesa review. Compos Part A 1997;28:903-22.

[13] Lomov Stepan V, Ivanov Dmitry S, Verpoest Ignaas, Zako Masaru, Kurashiki Tetsusei, Nakai Hiroaki, Hirosawa Satoru. Meso-FE modelling of textile composites: road map, data flow and algorithms. Compos Sci Technol 2007;67:67.

[14] Meza Lucas R, Schormans Jim MJ, Remmers Joris JC, Deshpande Vikram S. Shear response of 3D non-woven carbon fibre reinforced composites. J Mech Phys Solids 2019;125:276-97.

[15] Yang Zhengmao, Liu Hui. An elastic-plastic constitutive model for thermal shocked oxide/oxide ceramic-matrix composites. Int J Mech Sci 2020:105528. https://doi. org/10.1016/j.ijmecsci.2020.105528.

[16] Gao Xiguang, Dong Hongnian, Zhang Sheng, Song Yingdong. Multi-scale modeling and experimental study of the strength of plain-woven sic/sic composites. Appl 
Compos Mater 2019.

[17] Rao MP, Pantiuk M, Charalambides PG. Modeling the geometry of satin weave fabric composites. J Compos Mater 2008;43:19-56.

[18] Daggumati S, Van Paepegem W, Degrieck J, Xu J, Lomov SV, Verpoest I. Local damage in a 5-harness satin weave composite under static tension: Part II-meso-FE modelling. Compos Sci Technol 2010;70:1934-41.

[19] Zhang Daxu, Hayhurst DR. Prediction of stress-strain and fracture behaviour of an 8-Harness satin weave ceramic matrix composite. Int J Solids Struct 2014;51(21-22):3762-75.

[20] El Said Bassam, Ivanov Dmitry, Long Andrew C, Hallett Stephen R. Multi-scale modelling of strongly heterogeneous 3D composite structures using spatial voronoi tessellation. J Mech Phys Solids 2016;88:50-71.

[21] Ullah Z, Kaczmarczyk L, Pearce CJ. Three-dimensional nonlinear micro/meso-mechanical response of the fibre-reinforced polymer composites. Compos Struct 2017;161:204-14.

[22] Boise CM, Jack DA, Smith DE. A multiple anisotropic material finite element and its application to stiffness tensor predictions for woven composite lamina. Compos Part A 2017;99:208-20.

[23] Zhou Lichuan, Sun Xiaohao, Chen Mingwei, Zhu Yinbo, Hengan Wu. Multiscale modeling and theoretical prediction for the thermal conductivity of porous plainwoven carbonized silica/phenolic composites. Compos Struct 2019;215:278-88.

[24] Cairns Douglas S, Nelson Jared W, Woo Kyeongsik, Miller David. Progressive damage analysis and testing of composite laminates with fiber waves. Compos Part A 2016;90:51-61.

[25] Wang Liang, Wu Jiayi, Chen Chuanyong, Zheng Chuanxiang, Li Biao, Joshi Sunil Chandrakant, Zhou Kun. Progressive failure analysis of 2D woven composites at the meso-micro scale. Compos Struct 2017;178:395-405.

[26] Shi Yuan, Jain Neraj, Koch Dietmar. Investigation and modeling of tensile failure properties of wound ceramic matrix composites. Compos Part A 2018;114:316-26.

[27] Yang Zhengmao, Liu Hui. A continuum damage mechanics model for 2-D woven oxide/oxide ceramic matrix composites under cyclic thermal shocks. Ceram Int
2019;46:6029-37.

[28] Yang Zhengmao, Liu Hui. A continuum fatigue damage model for the cyclic thermal shocked ceramic-matrix composites. Int J Fatigue 2020;134:105507. https://doi. org/10.1016/j.ijfatigue.2020.105507.

[29] Moser B, Rossoll A, Weber L, Beffort O, Mortensen A. Nextel ${ }^{\mathrm{tm}} 610$ alumina fibre reinforced aluminium: influence of matrix and process on flow stress. Compos Part A 2001;32(8):1067-75.

[30] Moser B, Rossoll A, Weber L, Beffort O, Mortensen A. Damage evolution of Nextel $610^{\mathrm{tm}}$ alumina fibre reinforced aluminium. Acta Mater 2004;52(3):573-81.

[31] Yang Zhengmao, Yang Junjie. Investigation of long-term thermal aging-induced damage in oxide/oxide ceramic matrix composites. J Eur Ceram Soc 2020;40:1549-56.

[32] Pan N, Chen HC, Thompson J, Inglesby MK, Khatua S, Zhang XS, Zeronian SH. The size effects on the mechanical behaviour of fibres. J Mater Sci 1997;32:2677-85.

[33] Xiong Xiaoshuang, Hua Lin, Miao Menghe, Shen Shirley Z, Li Xiang, Wan Xiaojin, Guo Wei. Multi-scale constitutive modeling of natural fiber fabric reinforced composites. Compos Part A 2018;115:383-96.

[34] Liang Biao, Zhang Weizhao, Fenner Joel S, Gao Jiaying, Shi Yi, Zeng Danielle, Xuming Su, Liu Wing K, Cao Jian. Multi-scale modeling of mechanical behavior of cured woven textile composites accounting for the influence of yarn angle variation. Compos Part A 2019;124:105460.

[35] Ha Sung Kyu, Jin Kyo Kook, Huang Yuanchen. Micro-mechanics of failure (MMF) for continuous fiber reinforced composites. J Compos Mater 2008;42:1873-95.

[36] Gao Xiguang, Li Long, Song Yingdong. A temperature-dependent constitutive model for fiber-reinforced ceramic matrix composites and structural stress analysis. Int $\mathrm{J}$ Damage Mech 2014;23(4):507-22.

[37] Chamis Christos C. Mechanics of composite materials: past, present, and future. J Compos Technol Res 1989;11(1):3-14.

[38] Huang Zhengming. The mechanical properties of composites reinforced with woven and braided fabrics. Compos Sci Technol 2000;60(4):479-98. 